\title{
Growth of $\{110\}$-one-axis-oriented perovskite-type oxide films using self-aligned epitaxial (101)PdO //(111) Pd double layers
}

\author{
Hiroki Tanaka $^{1}$, Tetsuro Kariya ${ }^{1,2}$, Takao Shimizu ${ }^{1,3}$, Kiyoshi Uchiyama ${ }^{4}$, and \\ Hiroshi Funakubo ${ }^{1,3}$
}

${ }^{1}$ Department of Innovative and Engineered Material, Tokyo Institute of Technology, 4259 Nagatsuta-cho, Midori-ku, Yokohama 226-8502, Japan

${ }^{2}$ Sanyo Special Steel Co., Ltd, 3007, Nakashima, Shikama-ku, Himeji, Hyogo 672-8677, Japan

${ }^{3}$ Materials Research Center for Element Strategy, Tokyo Institute of Technology, 4259

Nagatsuta, Midori, Yokohama 226-8503, Japan

${ }^{4}$ Depaartment of Creative Engineering, National Institute of Technology, Tsuruoka College, 104 Inooka Sawada, Tsuruoka, Yamagata 997-8511, Japan

Corresponding author: Kiyoshi Uchiyamaｅmail: uchiyama@tsuruoka-nct.ac.jp

Department of Creative Engineering, National Institute of Technology, Tsuruoka

College, Inooka Sawada 104, Tsuruoka, Yamagata 997-8511, Japan

$+81-235-25-9097$ 


\section{$\underline{\text { Abstract }}$}

Self-aligned (101)-one-axis-oriented PdO layer were obtained on (111) Pd films prepared on (111) $\mathrm{Pt} / \mathrm{TiO}_{\mathrm{x}} / \mathrm{SiO}_{2} / \mathrm{Si}$ [abbreviated as (111) $\mathrm{Pt} / \mathrm{Si}$ ] substrates by the heat treatment at $750^{\circ} \mathrm{C}$ under atmospheric oxygen flow. Films with $(110)_{c}$-oriented $\mathrm{SrRuO}_{3}$ with perovskite structure was successfully deposited at $500{ }^{\circ} \mathrm{C}$ on a (101)-oriented PdO layer by an RF magnetron sputtering method due to their relatively small lattice mismatch.

A (101)-oriented $\operatorname{Sr}\left(\mathrm{Zr}_{0.8} \mathrm{Y}_{0.2}\right) \mathrm{O}_{3-\delta}(\mathrm{SZYO})$ film can be successfully prepared on $(110)_{c}$-oriented $\mathrm{SrRuO}_{3}$ and its proton conductivity is almost the same as that of $(111)_{c}$-oriented SZYO but slightly smaller than that of $(111)_{c}$-oriented one. As the conductivity is strongly affected by the film crystallinity, we can conclude that the newly fabricated $(110)_{\mathrm{c}}$-oriented SZYO has almost the same crystallinity comparing to the films with other orientation.

We have successfully demonstrated that the use of (101)PdO//(111)Pd double layer is a good candidate to grow $\{110\}$-one-axis-oriented perovskite thin films on $\mathrm{Si}$ substrates.

KEYWORDS: (110)-oriented perovskite thin film, (101)PdO//Pd double layer, 
$\{110\}$-one-axis-oriented $\operatorname{Sr}\left(\mathrm{Zr}_{0.8} \mathrm{Y}_{0.2}\right) \mathrm{O}_{3-\delta}$ 


\section{Introduction}

Perovskite-type oxide and their related structured oxide have been widely investigated due to their wide varieties of functionality including superconductivity, ferroelectricity, ferromagnetism, and so on [1]. Films for these materials have been also widely investigated for devise applications. Some of these properties are known to have a strong orientation dependency [2]. For example, spontaneous polarization of the ferroelectricity is appeared along polar axis and its crystal orientation dependency can be described by taking into account of tilting angles from the polar axis [3]. These orientation dependencies have been mainly investigated using epitaxial films grown on single crystal substrates with different orientation cuts, e.g. single crystal substrates of $\mathrm{SrTiO}_{3}$ (STO) with (100)-, (110)- and (111)-cuts. Particularly, \{100\} -oriented films have been widely investigated using various kinds of substrates [4]. On the other hand, $\{111\}$-oriented films is reported to have various advantage in view of the properties, such as the large relative dielectric constant of epitaxial grown $\mathrm{Pb}(\mathrm{Zr}, \mathrm{Ti}) \mathrm{O}_{3}(\mathrm{PZT})$ films [5]. Compared to these two directions, less number of researches on $\{110\}$-oriented films have been studied. However, there are some reports on good properties for $\{110\}$ -oriented perovskite films. For example, $\{110\}$-oriented tetragonal PZT thin films grown on the (110)STO substrates exhibit two $90^{\circ}$ domain structures less than three in 
typical epitaxial $\{100\}$-oriented PZT thin films. Reduction of the number in domain variants from three to two is pointed out to leads the minimization of domain wall pinning at the interfacial steps. As a result, the mobility of $90^{\circ}$ domain walls is enhanced and the piezoelectric properties of ferroelectric films are improved [6].

One reasons of less research activity on 1110 )-orientated films is difficulty of the growth of $\{110\}$-oriented films on widely used substrates, such as (100)Si. This is different from the $\{100\}$ - and $\{111\}$ - oriented films in which a various kinds of buffer layers to grow $\{100\}$ - and $\{111\}$ - orientations of perovskite-type structure have been reported [7-9]. For example, $\{100\}$ - and $\{111\}$-oriented films of $\mathrm{SrRuO}_{3}(\mathrm{SRO})$, one of the widely-used perovskite-type oxides with high conductivity [10], were successfully prepared on (111) $\mathrm{Pt} / \mathrm{TiO}_{\mathrm{x}} / \mathrm{SiO}_{2} /(100) \mathrm{Si}$ [here-in-after we abbreviated as (111)Pt/Si] substrates by using $(100)_{c} \mathrm{LaNiO}_{3}(\mathrm{LNO}) /(111) \mathrm{Pt}$ and (111)Pt buffer layers, respectively [11], where (111)Pt/Si substrates have been widely investigated for the Si-based devices applications that need bottom electrodes. This is because of the chemical and thermal stabilities of Pt and preferential (111)-orientation characteristics of Pt layer independent of the underlying substrates, especially prepared by RF magnetron sputtering method, where $\mathrm{TiO}_{\mathrm{x}}$ is used as an adhesion layer of Pt layer on oxidized Si substrates [12]. However, $\{110\}$-one-axis-oriented films with perovskite structure have been hardly 
reported on (111)Pt/Si substrates within the limit on our survey. In the present study, $(110)_{c}$-oriented $\mathrm{SrRuO}_{3}(\mathrm{SRO})$ films were prepared on (111)Pt/Si substrates using a self-aligned epitaxial (101)PdO//(111)Pd double layer. Using this double layer, we considered we can deposit high quality (110)-oriented perovskite thin films. Among various kinds of perovskites, we chose $\operatorname{Sr}\left(\mathrm{Zr}_{0.8} \mathrm{Y}_{0.2}\right) \mathrm{O}_{3-\delta}(\mathrm{SZYO})$, one of the proton conductor, for depositing (110)-oriented perovskites to reveal its crystallographic anisotropy of the proton conductivity.

Proton conductive perovskites, including SZYO, are considered to be one of the candidates of electrolytes to achieve solid oxide fuel cells (SOFCs) with an intermediate temperature (IT) $\left(400-600^{\circ} \mathrm{C}\right)$ operation, which is so-called as IT-SOFC [13-16]. Proton conductive oxide was discovered by Ishihara et al. [17] and was proposed for applying it to IT-SOFCs [13]. In the perovskite based proton conductive oxides, proton is transferred between oxygen sites by hopping, which bring proton conductivity at intermediate temperatures. However, those of proton conductive materials have been reported that they exhibit low conductivity [18-20] and improving proton conductivity is needed to improve IT-SOFC performances.

In general, proton conductivity has a crystallographic anisotropy because there might have the difference of the degree of difficulty of hopping among the 
crystallographic directions. However, few researches on the anisotropy of proton

conductivity were reported and we consider revealing the anisotropy is indispensable for revealing a mechanism of proton conductivity and improving them.

In this paper, the crystallographic anisotropy of proton conductivity of SZYO was investigated by using our new deposition technique for fabricating (110)-oriented perovskite thin films.

\section{Strategy of buffer layer for $\{110\}$-oriented perovskite films}

For the growth of $\{110\}$-oriented films of perovskite-type oxide on (111)Pt/Si substrates, two different lattice spacing must match along [100] and [101], e.g. $a$ and $\sqrt{2} a$ with the internal angle of $90^{\circ}$, where $a$ is the lattice parameter in case of cubic structure. This is different from the case of $\{100\}$ - and $\{111\}$ - orientated films because only one lattice matching must be taken into account.

In addition, it is also important that the buffer layers have similar crystal structure and lattice parameters with Pt to get high quality films in case of the growth on widely investigated Pt electrodes, such as (111)Pt/Si substrate. The noble metals such as palladium, iridium, and rhodium have same crystal structure of face center cubic $(f c c)$ with $\mathrm{Pt}$ and their lattice constant is close to that of Pt. Among these metals, palladium 
has the small lattice mismatch of $0.8 \%$ with $\mathrm{Pt}$ at room temperature. This imagines us the epitaxial growth of $\mathrm{Pd}$ on $\mathrm{Pt}$, (111)Pd//(111)Pt. Interesting point of Pd layer is that the oxidized $\mathrm{Pd}, \mathrm{PdO}$, is reported to have a epitaxial relationship with (101)-orientation on the (111) Pd surface as schematically shown in Fig. 1 [21, 22]. Lattice spacing of $\mathrm{PdO}$ along [101], $0.613 \mathrm{~nm}$, is similar to that of perovskite along [110], ranging from 0.549 to $0597 \mathrm{~nm}$ typically. This imagines us that the in-plane lattice spacing of $\mathrm{PdO}$ along [110] can be matched with those of perovskites. Therefore, it is expected that $\{110\}$-oriented perovskite-type oxides can be grown on the (101)-oriented $\mathrm{PdO}$ as schematically shown in Fig.1.

\section{Experimental procedure}

$\mathrm{Pt}, \mathrm{Pd}$, and SRO films with respective $100 \mathrm{~nm}, 100 \mathrm{~nm}$ and $50 \mathrm{~nm}$ in film thickness were prepared by RF magnetron sputtering method. Deposition temperature and the total pressure are as follows; $700^{\circ} \mathrm{C}$ and $2.67 \mathrm{~Pa}$ for $\mathrm{Pt}, 500^{\circ} \mathrm{C}$ and $2.67 \mathrm{~Pa}$ for $\mathrm{Pd}$, and $500^{\circ} \mathrm{C}$ and $26.7 \mathrm{~Pa}$ for $\mathrm{SRO}$. PdO films were oxidized $750^{\circ} \mathrm{C}$ with oxygen gas flow continuously after the Pd deposition in the sputtering chamber. Subsequently, $c a$. 50nm-thick SRO films with was deposited on the (101)PdO buffer layer as a bottom electrode. $3 \mu \mathrm{m}$-thick SZYO films were prepared on SRO films at $400^{\circ} \mathrm{C}$ by pulsed laser 
deposition (PLD) under a 6.7 $\mathrm{Pa} \mathrm{O}_{2}$ atmosphere. The composition of the SZYO film was assigned by the using wavelength dispersion $\mathrm{x}$-ray fluorescence spectrometer, calibrated with standard sample of sol-gel-derived SZYO thin films. The deposited SZYO by the rf-magnetron sputtering method is appeared to have $\mathrm{Sr} /(\mathrm{Zr}+\mathrm{Y})=0.5$ and $\mathrm{Zr} /(\mathrm{Zr}+\mathrm{Y})=0.8$, which is almost the same as that of the sputtering target.

The constituent phase and the degree of the out-of-plane and in-plane orientation of these films were characterized $\theta-2 \theta$ scan, and rocking curve scan and pole figure mapping, respectively using X-ray diffraction (PANalytical X'Pert MRD). A pseudocubic index was used as $(\mathrm{hkl})_{\mathrm{c}}$ for SRO.

Temperature dependency of the ionic conductivity for SZYO films was measured by AC impedance method with an impedance analyzer (Agilent 4194A) under humidified Ar atmosphere [23]. Ar gas was moisture at $40^{\circ} \mathrm{C}$, which generates about 70Pa of partial pressure of hydrogen. Stack structure of $\left(\mathrm{La}_{0.6} \mathrm{Sr}_{0.4}\right)\left(\mathrm{Co}_{0.2} \mathrm{Fe}_{0.8}\right) \mathrm{O}_{3-\delta}$ (LSCF)/ SZYO/SRO was used for the measurement, where LSCF top electrodes with a 1.5-mm in diameter were fabricated at $650^{\circ} \mathrm{C}$ on SZYO films by PLD method under a 6.7 $\mathrm{Pa}$ of $\mathrm{O}_{2}$ atmosphere.

\section{Results and discussion}


Figure 2 (a) shows XRD $\theta-2 \theta$ scan profile of $\mathrm{Pd}$ film prepared on (111)Pt/Si

substrate. As expected, (111)-preferred oriented Pd film was obtained on (111)-oriented Pt layer due to the small lattice mismatch. Figure 2 (b) shows $\theta-2 \theta$ XRD scan profile after the heat treatment. Only $\mathrm{PdO} 101$ diffraction peak was added on the profile, suggesting the (101) preferred orientation of $\mathrm{PdO}$ films as same as previously reported [21]. Fig. 2(c) show the rocking curve profile of PdO 101. Full width at half maximum $(F W H M)$ of $\mathrm{PdO} 101$ was $2.7^{\circ}$ that was wider than those of $\mathrm{Pd} 111$ and $\mathrm{Pt} 111,0.19^{\circ}$ and $0.16^{\circ}$, respectively. Figure $2(\mathrm{~d})$ shows the X-ray pole figure plot measured at $2 \theta=$ $34.0^{\circ}$ corresponding to $\mathrm{PdO} 101$. Concentrated ring-like spots were observed at azimuth angles, $p s i$, of $0^{\circ}, 60^{\circ}$, and $75^{\circ}$. These three ring-like spots suggest the growth of (101)-one-axis orientation of PdO layers on (111)-oriented Pd films by the simple oxidation process, self-alignment of (101)PdO on (111)Pd layer, (101)PdO//(111)Pd.

For the next step, an SRO thin film were prepared on the (101) $\mathrm{PdO} /(111) \mathrm{Pd} /(111) \mathrm{Pt} / \mathrm{Si}$ (hereafter described as (101) $\mathrm{PdO} / \mathrm{Si}$ ) substrate as well as depositing on the (111)Pt/Si and (100) ${ }_{c} \mathrm{LNO} /(111) \mathrm{Pt} / \mathrm{Si}$ (hereafter described as $\left.(100)_{c} \mathrm{LNO} / \mathrm{Si}\right)$ substrates for depositing $(110)_{c^{-}},(111)_{c^{-}}$and $(100)_{c^{-}}$-oriented SRO films, respectively. Figures 3(a) - (c) show the XRD $\theta-2 \theta$ scan profiles of the SRO films prepared at $500^{\circ} \mathrm{C}$ on each substrate. SRO $110 c$ and $200 c$ diffraction peaks were 
observed for the films prepared on (101) $\mathrm{PdO} / \mathrm{Si}$ and $(100)_{c} \mathrm{LNO} / \mathrm{Si}$, substrates as shown in Figs. 3 (b) and (c), respectively. On the other hand, noticeable peak from SRO film was not observed for the film on (111)Pt/Si substrate. Figures 3 (d)-(f) show the X-ray pole figure plots measured at fixed $2 \theta$ angle of $32.2^{\circ}$ corresponding to SRO $110 \mathrm{c}$. Ring-like peaks are observed at $\mathrm{psi}=35.3^{\circ}$ for $(111) \mathrm{c}$-oriented SRO layer, $\mathrm{Psi}=0^{\circ}$ and $60.0^{\circ}$ for $(110)$ c-oriented SRO layer, and $\mathrm{Psi}=45.0^{\circ}$ for $(100)_{c}$-oriented SRO layers as shown in Figs. 3 (d), (e) and (f), respectively. These results suggest that SRO films prepared on (111) Pt/Si, (101) $\mathrm{PdO} / \mathrm{Si}$ and $(100)_{c} \mathrm{LNO} / \mathrm{Si}$ substrates had perfectly $(111)_{\mathrm{c}^{-}}$, $(110)_{c^{-}}$and $(100)_{c^{-}}$one-axis orientations, respectively. This clearly suggests that self-aligned (101)PdO //(111)Pd double layers can grow (101)-oriented SRO films with perovskite structure. Rocking curve FWHM of SRO 110c and $100 \mathrm{c}$ were $2.6^{\circ}$ and $6.8^{\circ}$ for (110) and (100)-oriented SRO films, respectively. On the other hand, rocking curve FWHM of SRO $111_{c}$ was not separated from that of Pt for (111)c-oriented SRO films, because the lattice spacing of SRO $111 c$ and Pt 111 were almost the same.

To check the variety of the film quality of the perovskite films, proton conductor material of SZYO were prepared on these $(100)_{c^{-}},(110)_{c^{-}}$and $(111)_{c^{-}}$ one-axis oriented SRO films. Figures 4 (a)-(c) show $\theta$ - $2 \theta$ scans and X-ray pole figures measured at fixed $2 \theta$ angle of $30.8^{\circ}$, which corresponds to that of SZYO110, prepared 
on (a) $(111)_{c^{-}}$, (b) $(110)_{c^{-}}$, and (c) (100) $c_{c^{-}}$-oriented SRO films, respectively. Figures

(d)-(f) are the rocking curves of the samples. The $F W H M$ of rocking curves of

SZYO111, 110 and 100 were $1.7^{\circ}, 1.7^{\circ}$ and $6.8^{\circ}$, respectively. The wider $F W H M$ of the $\{100\}$-oriented SZYO film can be originated from the wider FWHMs of those of SRO and LNO peaks in the $\theta-2 \theta$ scans in fig. 3. The results shown in Fig. 4 clearly demonstrates that perovskite-type SZYO with (111)-, (110)- and (100)-one-axis orientations can be grown on widely-used (111)Pt/Si substrates by inserting (111) $\mathrm{C} S \mathrm{RO}$, (110)SRO/(110)PdO//Pd and (100) ${ }_{c} \mathrm{SRO} /(100) \mathrm{LNO}$ buffer layers.

Figure 5 (a) shows a typical result of cole-cole plot measurements for the $\{110\}$-oriented SZYO at $402^{\circ} \mathrm{C}$. We evaluated the electrolyte resistance by flitting assuming its equivalent circuit as in the Fig. 5(b), where CPE is a constant phase element and $\mathrm{Z}_{\mathrm{w}}$ is Warburg impedance.

Figure 6 shows Arrhenius plots of $\sigma \mathrm{T}$, where $\sigma$ and T are the ionic conductivity and the temperature, respectively, of $(100)_{c^{-}},(110)_{c^{-}}$, and $(111)_{c^{-}}$-oriented SZYO prepared on (111)Pt/Si substrates with buffer layers. All of the SZYO films show almost the same conductivity; however, $(100)_{c}$-oriented one is slightly larger than those of others with larger activation energy in the measured temperature region. A proton conductivity and its activation energy in polycrystalline film are presumed to be an 
average of ones in $(100)_{c^{-}},(110)_{c^{-}}$, and $(111)_{c^{-}}$direction and they are almost the same as that of the averaged conductivities in three directions [24]. In addition, the conductivity derived in this experiment was almost comparable to that of single crystal, which means these films possess good crystallinity which is comparable to that of single crystal [19]. As the conductivity is strongly affected by the crystallinity [25], the fabricated films in this study have almost the same crystallinity each other.

On the contrary, the activation energy of the conductivity showed the difference among the orientated SZYO films and was larger than that of the single crystal. The larger activation energy compared to that of the single crystal is considered to be reflecting the degree of difficulty of proton hopping because of grain boundaries. In the same way, we consider that the largest activation energy of (100)-oriented film is reflecting the anisotropy of difficulty of the proton hopping between oxygen sites.

As a consequence, we observed small difference of the conductivity among the orientated SZYO films; however, the anisotropy was not so large. We consider proton is small enough not to interrupt its hopping by the crystallographic configuration of ions. In addition, the conductivity of SZYO derived in this study is almost two decade smaller than that of doped BCO. Thus, we need further improvement in the conductivity to achieve good SOFC performance comparable to that of the SOFC using 
doped-BCYO as the electrolyte.

The results derived mentioned above demonstrate that $\mathrm{PdO} / / \mathrm{Pd}$ double layer worked as a good buffer layer to prepare $\{110\}$-oriented perovskite films on (111)Pt/Si substrates. It was already ascertained that $(111)_{c^{-}}$and $(100)_{c}$-preferred oriented films of SRO can be grown on wide variety of substrates including amorphous fused silica and metal foils using (111)Pt and (100) ${ }_{c} \mathrm{LNO} /(111) \mathrm{Pt}$ buffer layers [26] and [27]. Based on these results, (101)PdO//(111)Pd double layer prepared on (111)Pt is also expect to work as a buffer layer to prepare (110)-oriented perovskite films on Si substrate, which is widely used in thin film depositions.

\section{Summary}

$(110)_{c}$-oriented SRO and $\{110\}$-oriented SZYO/SRO were successfully grown on (111)Pt/Si substrates by inserting (101)PdO//(111)Pd double buffer layer. Prepared (110)-oriented SZYO films showed conducting properties similar to that of the (111)-oriented SZYO film; however, it is slightly smaller than that of the (100)-oriented one. In addition, the activation energy of the conductivity also showed small difference that the (100)-oriented one is larger than the others. The anisotropy observed in our study was not large; however, we consider it comes from the result that the degree of 
difficulty of proton hopping is almost the same in all any direction.

The result appeared in this paper show that (101)PdO//(111)Pd double layer

work as a good buffer layer for prepare (110)-oriented films of perovskite-type oxide on widely variety of substrates.

\section{Acknowledgements}

This research is partially supported by ALCA and A-STEP from Japan Science and Technology Agency (JST). This research is also partially granted by the Japan Society for the Promotion of Science (JSPS) through the "Funding Program for World-Leading Innovative R\&D on Science and Technology (FIRST Program)," initiated by the Council for Science and Technology Policy (CSTP). 


\section{References}

[1] W. Prellier, M. P. Singh, and P. Murugavel, The single-phase multiferroic oxides:

from bulk to thin film, J. Phys. Cond.. Matter 17 (2005) R803-R832.

[2] N. Izyumskaya, Y.-I. Alivov, S.-J. Cho, H. Morkoç, H. Lee, and Y.-S. Kang,

Processing, Structure, Properties, and Applications of PZT Thin Films, Crit. Rev. Solid

State Mater. Sci. 32 (2007) 111- 202.

[3] Y. Ehara, S. Utsugi, M. Nakajima, T. Yamada, T. Iijima, H. Taniguchi, M. Itoh, and

H. Funakubo, Spontaneous polarization estimation from the soft mode in strain-free

epitaxial polar axis-oriented $\mathrm{Pb}(\mathrm{Zr}, \mathrm{Ti}) \mathrm{O}_{3}$ thick films with tetragonal symmetry, Appl.

Phys. Lett. 98 (2011) 141914_1-3.

[4] B. A. Turtle, J. A. Voigt, T. J. Garino, R. W. Schwartz, D. L. Lamppa, and J. Thomas,

Highly Oriented, Chemically Prepared $\mathrm{Pb}(\mathrm{Zr}, \mathrm{Ti}) \mathrm{O}_{3}$ Thin Films, J. Amer. Ceram. Soc.

76 (1993) 1537-1544.

[5] T. Oikawa, M. Aratani, H. Funakubo, K. Saito, and M. Mizuhira, Composition and orientation dependence of electrical properties of epitaxial $\mathrm{Pb}\left(\mathrm{Zr}_{\mathrm{x}} \mathrm{Ti}_{1-\mathrm{x}}\right) \mathrm{O}_{3}$ thin films grown using metalorganic chemical vapor deposition, J. Appl. Phys. 95 (2004)

$3111-3115$.

[6] J. Ouyang, J. Slusker, I. Levin, D.-M. Kim, C.-B. Eom, R. Ramesh, and L. Roytburd, 
Engineering of Self-Assembled Domain Architectures with Ultra-high Piezoelectric Response in Epitaxial Ferroelectric Films, Adv. Funct. Mater. 17 (2007) 2094-2100.

[7] C.-C. Yang, M.-S. Chen, T.-J. Hong, C.-M. Wu, J.-M. Wu, and T.-B. Wu, Preparation of (100) - oriented metallic $\mathrm{LaNiO}_{3}$ thin films on Si substrates by radio frequency magnetron sputtering for the growth of textured $\mathrm{Pb}\left(\mathrm{Zr}_{0.53} \mathrm{Ti}_{0.47}\right) \mathrm{O}_{3}$, Appl. Phys. Lett. 66 (1995) 2643-2645.

[8] H. Miyazaki, T. Goto, Y. Miwa, T. Ohno, H. Suzuki, T. Ota, and M. Takahashi, Preparation and evaluation of $\mathrm{LaNiO}_{3}$ thin film electrode with chemical solution deposition, J. Eur. Ceram. Soc. 24 (2004) 1005-1008.

[9] G. R. Fox, S. Trolier-Mckinstry, and S.B. Krupanidhi, and L. M. Casas, $\mathrm{Pt} / \mathrm{Ti} / \mathrm{SiO}_{2} / \mathrm{Si}$ substrates, J. Mater. Res. 10 (1995) 1508-1515.

[10] C. B. Eom, R. J. Cava, R. M. Fleming, J. M. Phillips, R. B. vanDover, J. H. Marshall, J. W. P. Hsu, J. J. Krajewski, and W. F. Peck, Jr., Single-Crystal Epitaxial Thin Films of the Isotropic Metallic Oxides $\mathrm{Sr}_{1-\mathrm{x}} \mathrm{Ca}_{\mathrm{x}} \mathrm{RuO}_{3}(0 \leq \mathrm{x} \leq 1)$, Science. 258 (1992) 1766-1769.

[11] S. Okamoto, S. Yokoyama, Y. Honda, G. Asano, and H. Funakubo, Crystal Orientation Dependence on Electrical Properties of $\mathrm{Pb}(\mathrm{Zr}, \mathrm{Ti}) \mathrm{O}_{3}$ Thick Films Grown on Si Substrates by Metalorganic Chemical Vapor Deposition, Jpn. J. Appl. Phys. 43 (2004) 
$6567-6570$.

[12] U. Schmid, The impact of thermal annealing and adhesion film thickness on the resistivity and the agglomeration behavior of titanium/platinum thin films, J. Appl. Phys. 103 (2008) 054902_1-12.

[13] H. Iwahara, T. Yajima, T. Hibino, and H. Ushida, Performance of Solid Oxide Fuel

Cell Using Proton and Oxide Ion Mixed Conductors Based on $\mathrm{BaCe}_{1-\mathrm{x}} \mathrm{Sm}_{\mathrm{x}} \mathrm{O}_{3-\alpha}, \mathrm{J}$.

Electrochem. Soc. 140 (1993) 1687-1691.

[14] T. Shimada, C. Wen, N. Taniguchi, J. Otomo, and H. Takahashi, The high temperature proton conductor $\mathrm{BaZr}_{0.4} \mathrm{Ce}_{0.4} \mathrm{In}_{0.2} \mathrm{O}_{3-\alpha}$, J.Power Sources 131 (2004) 289-292.

[15] N. Ito, M. Iijima, K. Kimura and S. Iguchi, New intermediate temperature fuel cell with ultra-thin proton conductor electrolyte, J. Power Sources 152 (2005) 200-203.

[16] K. Uchiyama, Y. Isse, Y. Hori, T. Nishida, Y. Uraoka, T. Kariya and K. Yanagimoto, Structural and electrical characterization of Y-doped $\mathrm{SrZrO}_{3}$ thin films for solid oxide fuel cells, Proc. 6th Thin-Films Materials and Devices Meeting (Kyoto) (2010), pp. $100228102 \_1-4$.

[17] H. Iwahara, T. Esaka, H. Uchida, and N. Maeda, Proton conduction in sintered oxides and its application to steam electrolysis for hydrogen production, Solid State 
Ionics 3-4 (1981) 359-363.

[18] S. Shin, H. H. Huang, M. Ishigame, and H. Iwahara, Protonic conduction in the single crystals of $\mathrm{SrZrO}_{3}$ and $\mathrm{SrCeO}_{3}$ doped with $\mathrm{Y}_{2} \mathrm{O}_{3}$, Solid State Ionics 40-41 (1990) 910-913.

[19] T. Higuchi, T. Tsukamoto, N. Sata, K. Hiramoto, M. Ishigame, and S. Shin, Protonic Conduction in the Single Crystal of $\mathrm{SrZr}_{0.95} \mathrm{M}_{0.05} \mathrm{O}_{3}(\mathrm{M}=\mathrm{Y}, \mathrm{Sc}, \mathrm{Yb}, \mathrm{Er})$, Jpn. J. Appl. Phys. 40 (2001) 4162-4163.

[20]T. Higuchi, T. Tsukamoto, S. Yamaguchi, N. Sata, K. Hiramoto, M. Ishigame, and S. Shin, Protonic Conduction in the Single Crystal of Sc-Doped $\mathrm{SrZrO}_{3}$, Jpn. J. Appl. Phys. 41 (2002) 6440-6442.

[21] H. H. Kan and J. F. Weaver, $\mathrm{A} \operatorname{PdO}\left(\begin{array}{lll}1 & 0 & 1\end{array}\right)$ thin film grown on $\operatorname{Pd}\left(\begin{array}{lll}1 & 1 & 1\end{array}\right)$ in ultrahigh vacuum, Surf. Sci. 602 (2008) L53-L57.

[22] J. F. Weaver, S. P. Devarajan, and C. Hakanoglu, Facile C-H Bond Cleavage and Deep Oxidation of Propane on a PdO(101) Thin Film, J. Phys. Chem. C 113 (2009) 9773-9782.

[23] K. D. Kreuer, Proton -Conducting Oxides, Annu. Rev. Mater. Res. 33 (2003) 333-359.

[24] M. Arab Pour Yazdi, P. Briois, S. Georges, and A. Billard, Electrical and structural 
investigations of perovskite structure reactively sputter deposited coatings, Solid State Ionics 180, (2009) 1246-1251.

[25] D. Pergolesi, E. Fabbri, A. D’Epifanio, E. Di Bartolomeo, A. Tebano, S. Sanna, S. Licoccia, G. Balestrino, and E. Traversa, High proton conduction in grain-boundary-free yttrium-doped barium zirconate films grown by pulsed laser deposition, Nat. Mater. 9 (2010) 846-852.

[26] S. Ito, H. Funakubo, I. P. Koutsaroff, M. Zelner, and A. Cervin-Lawry, Effect of the thermal expansion matching on the dielectric tunability of (100)-one-axis-oriented $\left(\mathrm{Ba}_{0.5} \mathrm{Sr}_{0.5}\right) \mathrm{TiO}_{3}$ thin films, Appl. Phys. Lett. 90 (2007) 142910_1-3.

[27] T. Shiraishi, H. Einishi, S. Yasui, H. Funakubo, T. Hasegawa, M. Kurosawa, M. Ishikawa, H. Uchida, and Y. Sakashita, Ferroelectric and piezoelectric properties of (K,Na) $\mathrm{NbO}_{3}$ thick films prepared on metal substrates by hydrothermal method, J. Korean Phys. Soc. 62 (2013) 1055-1059. 


\section{Figure captions}

FIG.1 Schematic drawings of (a) $\{110\}$-oriented perovskite-type oxides layer, (b)

(101) PdO layer and (c) (111)Pd layer on (111)Pt/Si substrate.

FIG.2 XRD $\theta-2 \theta$ patterns of (a) Pd film prepared on (111)Pt/Si substrate and (b)

heat-treated Pd film prepared on (111)Pt/Si substrate, and the (c) rocking curve scan of

$\mathrm{PdO} 101$, and (d) X-ray pole figure plots measured at fixe $\mathrm{d} 2 \theta$ angle corresponding to

$\mathrm{PdO} 101$ for the heat-treated Pd films prepared on (111)Pt/Si substrate .

FIG.3 XRD patterns and X-ray pole figure plots of SRO films prepared on (a)

(111) $\mathrm{Pt} / \mathrm{Si}$, (b) (101) $\mathrm{PdO} / \mathrm{Si}$ and (c) (100) ${ }_{c} \mathrm{LNO} / \mathrm{Si}$ substrates. The pole figure was measured at fixed $2 \theta$ angle of $32.2^{\circ}$, which corresponds to SRO $110 c$.

FIG.4 XRD patterns and X-ray pole figure plots of SZYO films prepared on (a)

$(111)_{c^{-}},(\mathrm{b})(110)_{c^{-}}$, and (c) $(100)_{c^{-}}$-oriented SRO. The pole figure was measured at fixed $2 \theta$ angle of $30.8^{\circ}$, which corresponds to SZYO 110. Rocking curves of SZYO films deposited on one-axis-oriented SRO films are also shown in Figs. 4 (d)-(f). 
FIG.5 (a) Typical cole-cole plot of the samples, (b) the equivalent circuit which was used for electrolyte resistance analysis.

FIG.6 Arrhenius plots of ionic conductivity for $\{100\},\{110\}$, and $\{111\}$-oriented SZYO films prepared on (111)Pt/Si substrates. Reported data for polycrystalline SZYO with composition of $\mathrm{Y} /(\mathrm{Zr}+\mathrm{Y})=0.16$ prepared by $\mathrm{RF}$ magnetron sputtering method is also shown in this figure [16]. 
Fig.1

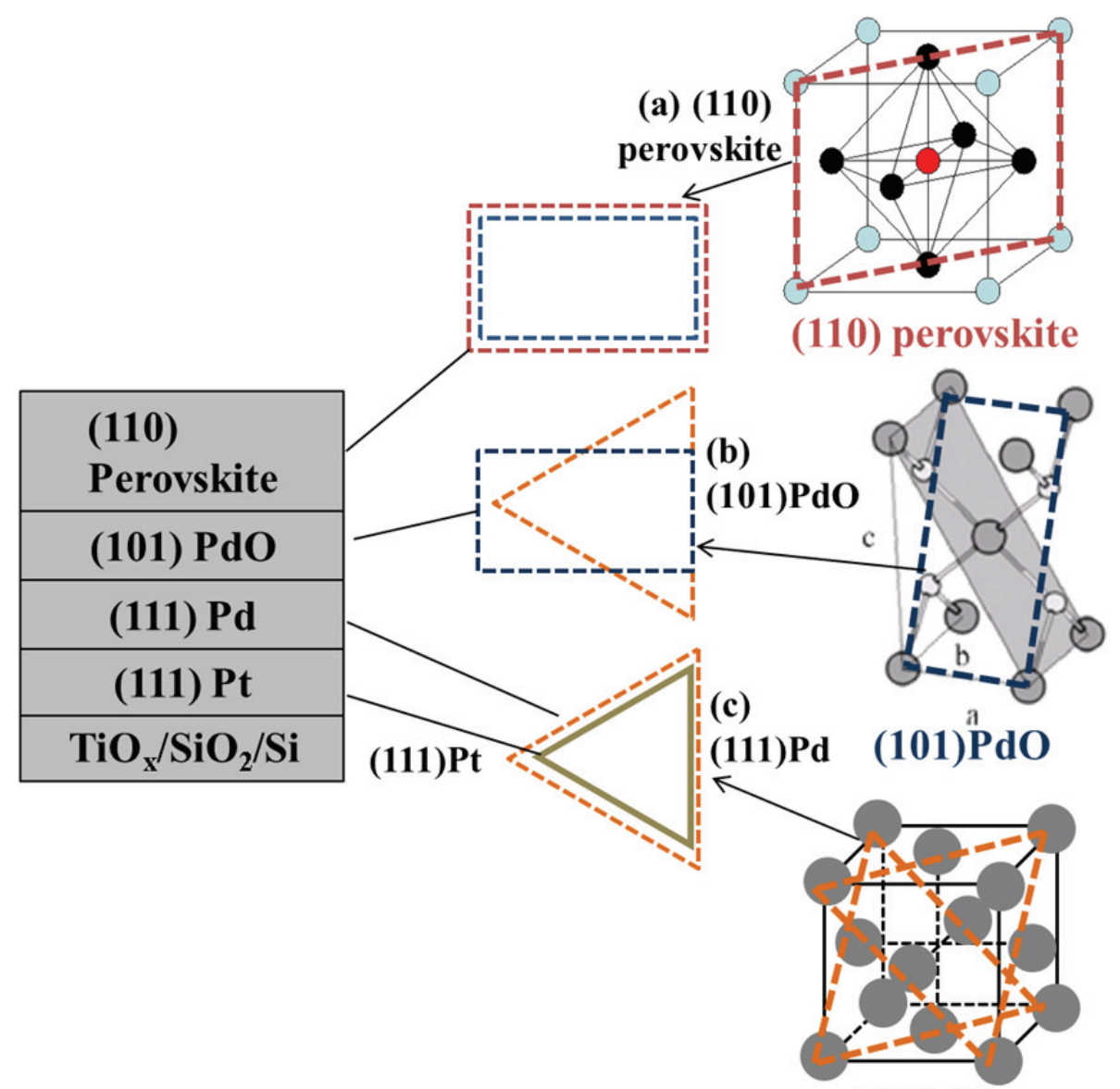

(111) Pd 
Fig.2

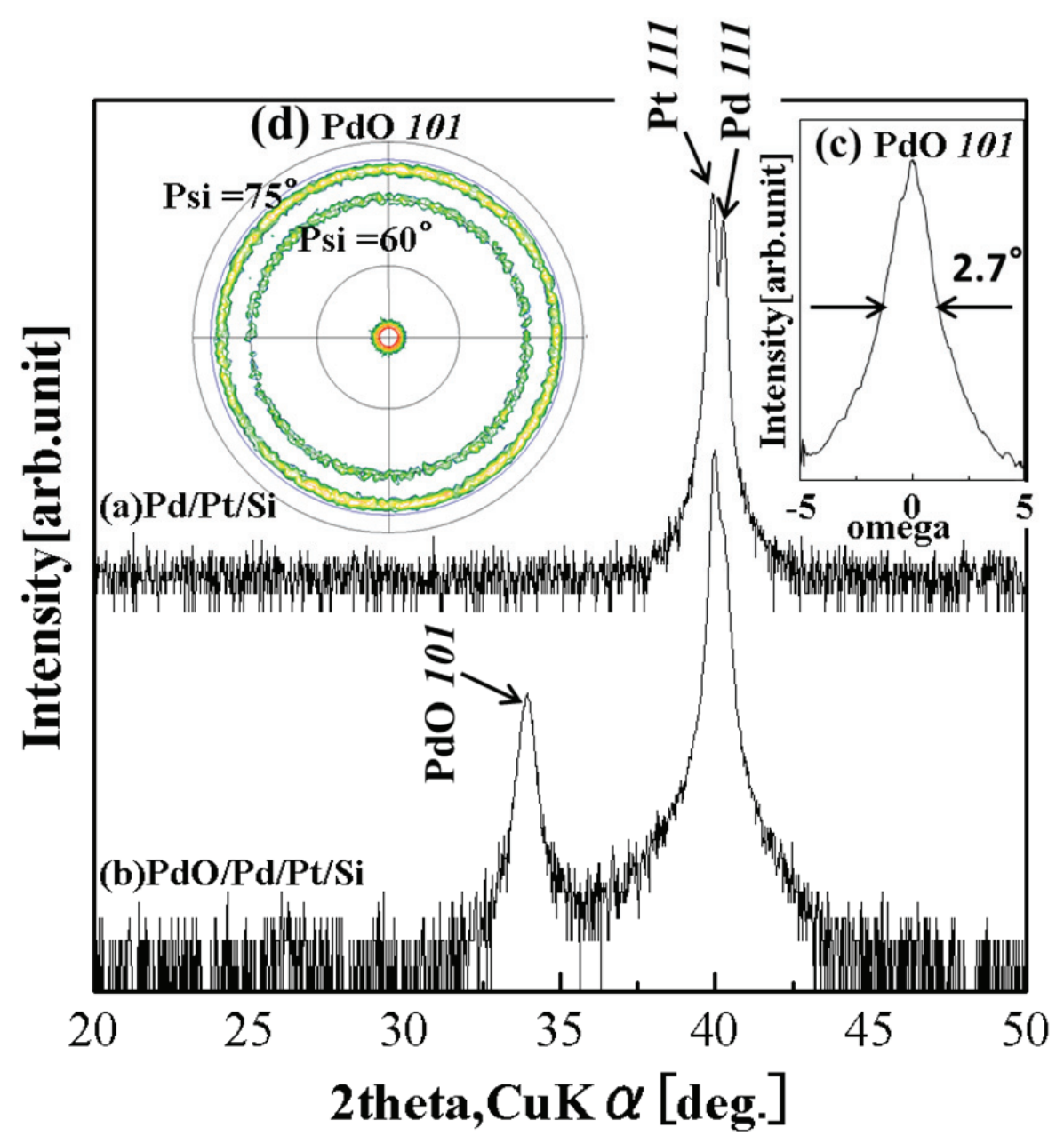


Fig.3

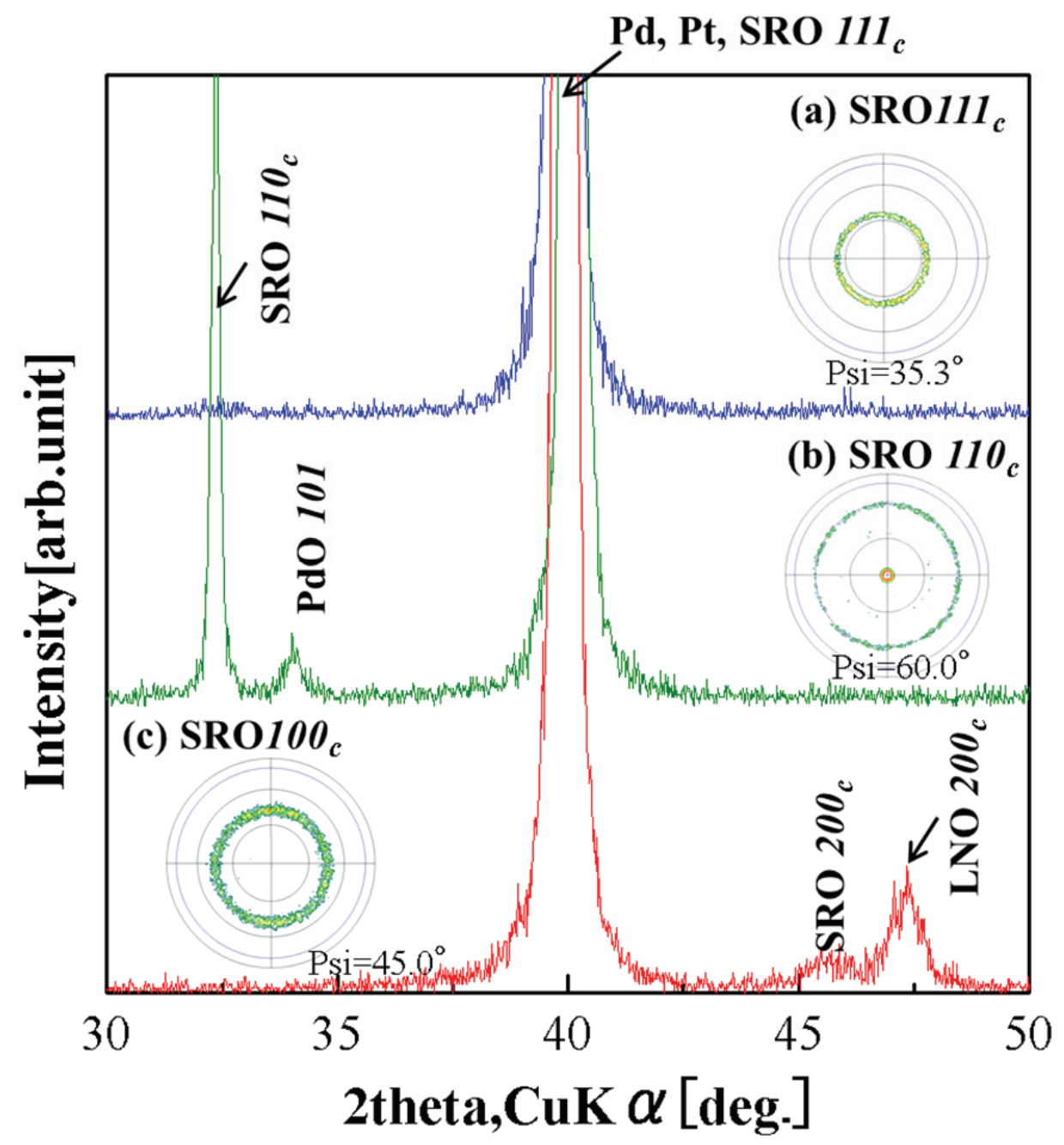


Fig.4

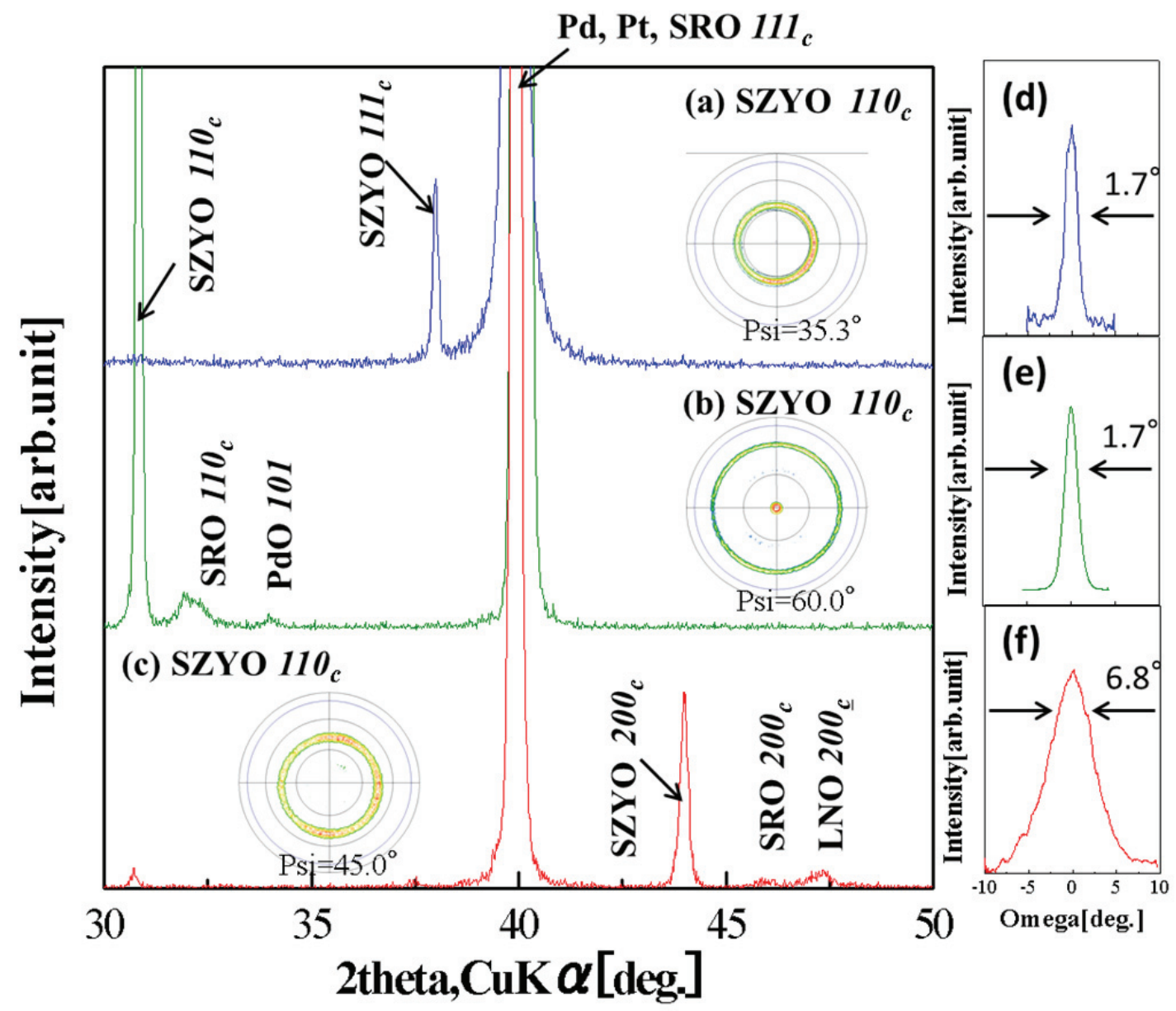


Fig.5

(a)

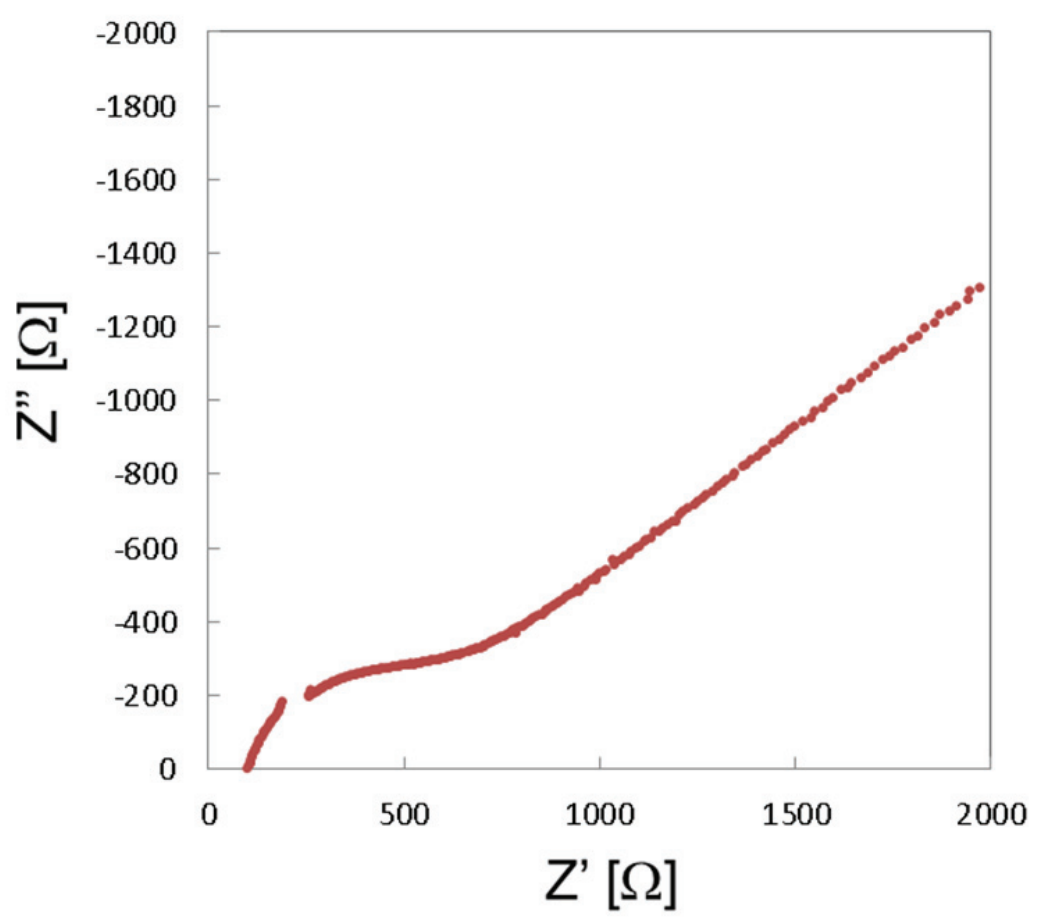

(b)

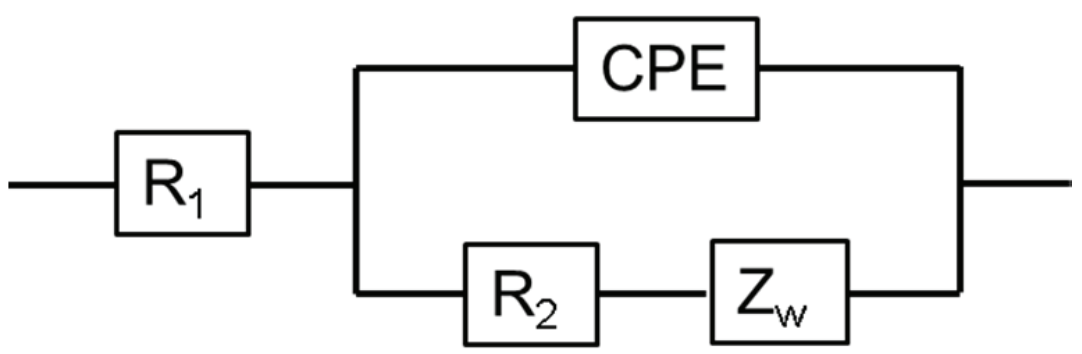

$R_{1}$ : electrode resistance

$\mathrm{R}_{2}$ : electrolyte resistance

CPE: constant phase element

$\mathrm{Z}_{\mathrm{w}}$ : Warburg impedance 
Fig.6

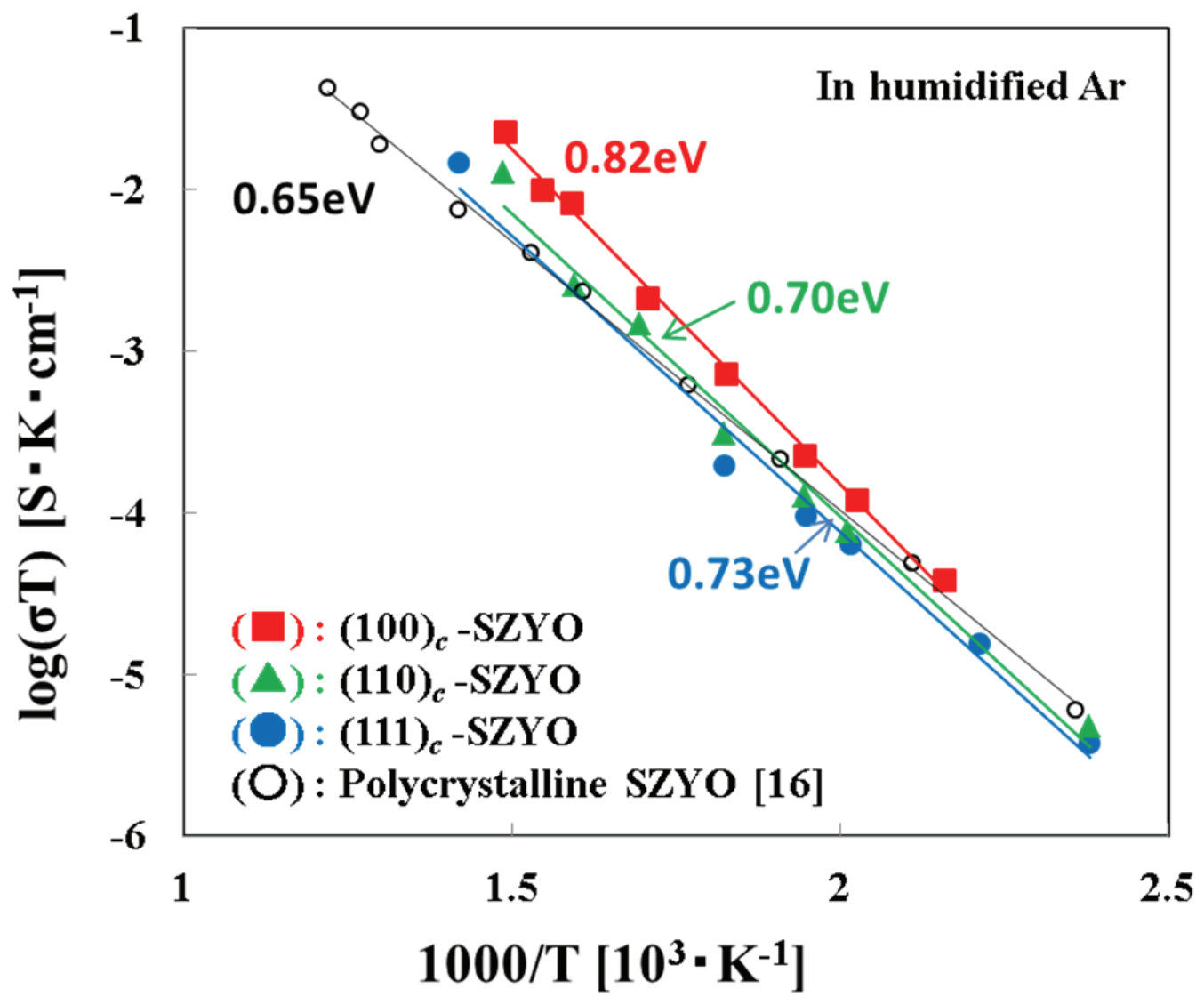

